\title{
Contributions of symptomatic osteoarthritis and physical function to incident cardiovascular disease
}

\author{
Michela Corsi ', Carolina Alvarez ${ }^{2}$, Leigh F. Callahan²,3, Rebecca J. Cleveland ${ }^{2,3}$, Yvonne M. Golightly ${ }^{2,4,5}$,
} Joanne M. Jordan²,3, Amanda E. Nelson ${ }^{2,3}$, Jordan Renner ${ }^{2,6}$, Allen Tsai ${ }^{1}$ and Kelli D. Allen ${ }^{2,3,7^{*}}$

\begin{abstract}
Background: Osteoarthritis $(\mathrm{OA})$ is associated with worsening physical function and a high prevalence of comorbid health conditions. In particular, cardiovascular disease (CVD) risk is higher in individuals with OA than the general population. Limitations in physical function may be one pathway to the development of CVD among individuals with OA. This study evaluated associations of symptomatic knee OA (sxKOA), baseline physical function and worsening of function over time with self-reported incident CVD in a community-based cohort.

Methods: Our sample consisted of individuals from the Johnston County Osteoarthritis Project who did not report having CVD at baseline. Variables used to evaluate physical function were the Health Assessment Questionnaire (HAQ), time to complete 5 chair stands, and the 8 -ft walk. Worsening function for these variables was defined based on previous literature and cutoffs from our sample. Logistic regression analyses examined associations of sxKOA, baseline function and worsening of function over time with self-reported incident CVD, unadjusted and adjusted for relevant demographic and clinical characteristics.

Results: Among 1709 participants included in these analyses, the mean age was $59.5 \pm 9.5$ years, 63.6\% were women, $15 \%$ had sxKOA, and the follow up time was $5.9 \pm 1.2$ years. About a third of participants reported worsening HAQ score, about two-fifths had worsened chair stand time, half had worsened walking speed during the 8-ft walk, and 16\% self-reported incident CVD. In unadjusted analyses, sXKOA, baseline function, and worsening function were all associated with self-reported incident CVD. In multivariable models including all of these variables, sXKOA was not associated with incident CVD, but worsening function was significantly associated with increased CVD risk, for all three functional measures: $\mathrm{HAQ}$ odds ratio $(\mathrm{OR})=2.49$ (95\% confidence interval $(\mathrm{Cl}) 1.90-3.25)$, chair stands $\mathrm{OR}=1.58$ (95\% Cl 1.20-2.08), 8-ft walk OR=1.53 (95\%Cl 1.15-2.04). These associations for worsening function remained in models additionally adjusted for demographic and clinical characteristics related to CVD risk.
\end{abstract}

Conclusions: The association between symptomatic knee osteoarthritis and cardiovascular disease risk was explained by measures of physical function. This highlights the importance of physical activity and other strategies to prevent functional loss among individuals with symptomatic knee osteoarthritis.

Keywords: Osteoarthritis, Function, Cardiovascular disease

\footnotetext{
* Correspondence: kdallen@email.unc.edu

${ }^{2}$ Thurston Arthritis Research Center, University of North Carolina at Chapel Hill, 3300 Thurston Bldg., CB\# 7280, Chapel Hill, NC 27599, USA

${ }^{3}$ Department of Medicine, University of North Carolina at Chapel Hill, 125

MacNider Hall CB\# 7005, Chapel Hill, NC 27599, USA

Full list of author information is available at the end of the article
}

(c) The Author(s). 2018 Open Access This article is distributed under the terms of the Creative Commons Attribution 4.0 International License (http://creativecommons.org/licenses/by/4.0/), which permits unrestricted use, distribution, and reproduction in any medium, provided you give appropriate credit to the original author(s) and the source, provide a link to the Creative Commons license, and indicate if changes were made. The Creative Commons Public Domain Dedication waiver (http://creativecommons.org/publicdomain/zero/1.0/) applies to the data made available in this article, unless otherwise stated. 


\section{Background}

Osteoarthritis (OA) is a key contributor to functional disability that is becoming increasingly prevalent worldwide [1]. Symptomatic knee OA (sxKOA) is associated with functional limitations, which tend to worsen over time [2, 3]. Individuals with $\mathrm{OA}$ also have a significantly increased risk of cardiovascular disease (CVD) [4-6]. Individuals with OA tend to have multiple risk factors for CVD, including increased body mass index (BMI), hypertension, physical inactivity, and nonsteroidal anti-inflammatory drug (NSAID) use [5, 7]. For this reason, many hypotheses have been proposed regarding underlying pathophysiological mechanisms connecting OA and CVD, including the role of common molecular or metabolic pathways, chronic low-grade inflammation leading to both conditions, and the development of functional limitations from OA that in turn leads to a lack of physical activity, exacerbating both conditions $[7,8]$.

Recently, a number of studies have shown an association between physical function and CVD among individuals with OA. Schieir et al. showed that there was a greater risk of CVD in women with arthritis (with participants primarily having OA), compared to women without arthritis; the risk of CVD was further increased in women with both arthritis and physical limitations [9]. Among men in this study, there was only an increased risk of incident CVD for those who reported both arthritis and physical limitations. Together these results suggest that physical function may play a significant role in the development of CVD in patients with arthritis. While this study focused broadly on arthritis, another cohort study found that the relationship between sxKOA and CVD was sustained when controlling for age, obesity, and metabolic factors, yet became insignificant when controlling for functional limitations [10]. However, this was a cross-sectional study, so a causal relationship could not be established. Another cohort study found that individuals with hip or knee OA who used a walking aid due to functional disability had a $30 \%$ greater risk of developing CVD than those who did not use a walking aid [11]. Another recent longitudinal cohort study found a dose-response relationship between the number of joints with OA and CVD risk; however, this relationship became non-significant when controlling for difficulty walking [12].

The purpose of this study was to examine associations of sxKOA, baseline physical function and worsening of function over time with self-reported incident CVD in a community-based cohort. In particular, we were interested in understanding whether different measures of physical function explained any relationship between sxKOA and CVD risk. This study adds to the literature in several important ways. First, it is one of few studies to examine the association between OA and CVD risk in a longitudinal analysis. Second, this study has multiple measures of function, including performance-based measures, which to our knowledge have not been used in other longitudinal studies of this topic. This deepens our understanding of how various functional measures may serve as predictors of CVD among individuals with OA. Third, this study examined not only baseline function but also change in function over time; prior studies have not assessed the role of worsening function over time and how this may play in the development of CVD in individuals with OA.

\section{Methods \\ Participants}

This study involved participants in the Johnston County Osteoarthritis Project (JoCo OA), an ongoing communitybased study focusing on hip and knee OA in a rural population [13]. Participants were civilian, non-institutionalized African-American and Caucasian adults aged 45 years and older selected from six townships within Johnston County, North Carolina. Initial enrollment occurred from 1991 to 1997 (Original Cohort), with first follow-up of this cohort occurring from 1999 to 2003. A second wave of enrollment occurred in 2003-2004 (Enrichment Cohort), aimed at enriching the sample for AA and younger individuals. First follow-up of this group occurred from 2006 to 2011. This research was reviewed and approved by the Institutional Review Board of the University of North Carolina, Chapel Hill; all participants provided written informed consent.

From participants enrolled in the Original Cohort $(N=3249)$ and Enrichment Cohort $(N=1141)$, we excluded individuals who did not have baseline clinic data, follow-up clinic data (due to loss to follow-up), and baseline and follow-up knee OA and CVD status. Then those who self-reported having CVD at baseline were excluded (Fig. 1). Finally, we excluded individuals who were missing baseline or follow-up data for functional tests or covariates, leading to a final sample size of 1709 . Complete case analysis (CCA) was used so that only participants with non-missing baseline covariates and physical function status at baseline and follow-up were analyzed. This proportion of participant with missing baseline or follow-up data for analyses was $4.9 \%$, so the impact of bias from their removal is likely to be small and CCA can be conducted regardless of the missing data pattern (Fig. 1) [14].

\section{Measures}

\section{Outcome: Incident self-report CVD}

Incident CVD was assessed at first follow-up through self-report. The definition of CVD was based on the World Health Organization criteria; due to changes in these criteria, self-report items differed slightly at different time points. For the Original Cohort, CVD at first follow-up was defined as having a heart attack, stroke, circulation or other heart problem. For the Enrichment 
$\mathrm{OA}=$ Osteoarthritis

$\mathrm{CVD}=$ Cardiovascular disease

$\mathrm{HAQ}=$ Health Assessment Questionnaire

NSAIDs $=$ Non-steroidal anti-

inflammatory drugs

$\mathrm{BMI}=$ Body Mass Index
Enrichment Cohort with Clinic Visit $(\mathrm{N}=1006)$
Baseline Sample ( $\mathrm{N}=4177)$

Complete Follow-Up Clinic

Visit ( $N=2243)$

Complete Data on Knee OA

and CVD Status ( $\mathrm{N}=2204)$

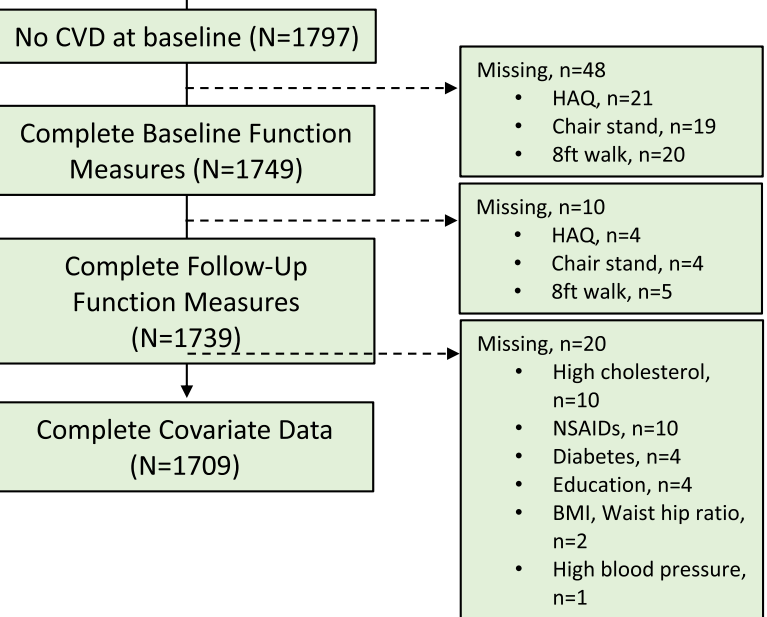

Fig. 1 Flow Chart of Participants Included in Analyses

Cohort, the incident CVD definition at first follow-up was expanded to include angina and congestive heart failure.

\section{Symptomatic knee $O A$}

JoCo OA participants had anteroposterior (Original Cohort enrollment) or posteroanterior (Enrhichment Cohort enrollment) radiographs taken of both knees while weight-bearing using a Synaflexer ${ }^{\circ}$ positioning device. All radiographs were read for Kellgren-Lawrence (K-L) score by a single bone and joint radiologist (JBR) without regard to participant's clinical status. Intrarater reliability and interrater reliability, assessed with another trained radiologist, were both high (weighted kappas were 0.89 and 0.86 , respectively). For the purpose of this study, radiographic $\mathrm{KOA}$ was defined as a K-L grade $\geq 2$. To assess joint symptoms, participants were asked: "On most days, do you have pain, aching, or stiffness in your...right/left knee." Participants responding "yes" to this question for a joint with radiographic OA were considered to have sxKOA.

\section{Function measures}

We included three measures of physical function: chair stands, 8-ft walk, and the Health Assessment Questionnaire Disability Index (HAQ).
Health assessment questionnaire The HAQ is a measure of self-reported disability, assessing ability to perform typical eight daily tasks (dressing, arising, eating, walking, reaching, gripping, chores, and hygiene) during the past 7 days [15]. Answers for each question are scored from 0 to 3, with 0 being no disability and 3 being complete disability. Per scoring guidelines, mean HAQ score was calculated for each participant if six or more of the eight categories were non-missing. We categorized baseline mean $\mathrm{HAQ}$ as follows: $\mathrm{HAQ}=0$, $0<\mathrm{HAQ}<1$, or $\mathrm{HAQ} \geq 1$, based on definitions previously used with JoCo OA data [16]. Based on the previously established minimum clinically important difference in HAQ score $[15,17,18]$, we defined a clinically significant change as baseline $+/-0.22$; individuals whose baseline scores rose by 0.22 or more were classified as worsening, and those whose baseline scores fell by 0.22 or more were classified as improving. If change in speed did not meet these values, participants were classified as having stayed the same. The groups were then dichotomized into improved/stayed the same, or worsened.

Chair stands Based on previously established protocols $[19,20]$, we assessed time for participants to complete 5 chair stands. Participants were seated in a chair with feet 
touching the floor and asked to rise without the use of arms as support. To ensure this, participants were asked to cross their arms at their wrists and hold them tight to their chests throughout the test. Participants who were unable to rise from a chair by themselves or scoot forward or stand up without using their arms were classified being unable to complete this test. For those able to perform this task, time taken to complete the 5 chair stands was recorded in seconds. We then categorized times into quartiles, based on baseline scores of those without sxKOA in our study sample. These thresholds were also determined separately for males and females due to evidence of differences in performance between these two groups [21]. For males, these cutoffs were time $<8.4 \mathrm{~s}$ (quartile 1, Q1), 8.4 $\mathrm{s}<$ time $<10.2 \mathrm{~s}$ (quartile 2, Q2), $10.2 \mathrm{~s}<$ time $<12.9 \mathrm{~s}$ (quartile 3, Q3), $12.9 \mathrm{~s}<$ time (quartile 4, Q4), or unable to complete all five chair stands. For females, these cutoffs were time $<9.0 \mathrm{~s}$ (Q1), $9.0 \mathrm{~s}<$ time $<11.3 \mathrm{~s}(\mathrm{Q} 2), 11.3 \mathrm{~s}<$ time $<14.1 \mathrm{~s} \quad(\mathrm{Q} 3)$, $14.1 \mathrm{~s}<$ time $(\mathrm{Q} 4)$, or unable to complete all five chair stands. We then categorized participants as either having worsened (moved up a quartile from baseline) or stayed the same / improved (remained in the same quartile or moved down a quartile from baseline) at the time of first follow-up. Participants unable to complete the chair stands at baseline were categorized as follows: staying the same / improving if they became able to complete at follow-up and worsening if they remained unable to complete at follow-up.

8-foot walk Using previously established procedures $[20,22]$, participants were asked to perform two trials in which they walked the $8-\mathrm{ft}$ at their normal pace; times of the two trials were averaged and converted to gait speed $(\mathrm{m} / \mathrm{s})$ and kept continuous for the baseline measure. We categorized participants as either worsening or staying the same/improving in gait speed at follow-up. We defined worsening as a decrease of $0.1 \mathrm{~m} / \mathrm{s}$ based on previous literature suggesting this may be a clinically relevant decline [23, 24].

Covariates Variables that could potentially confound the associations between sxKOA, function and CVD were included in multivariable models. These included: baseline enrollment group (Original vs. Enrichment cohort), age, gender, race (African American vs. Caucasian), education ( $<$ high school vs. $\geq$ high school), body mass index (BMI), waist to hip ratio (WHR), self-reported presence of diabetes, hypertension, or high cholesterol, and self-reported nonsteroidal anti-inflammatory drugs (NSAIDs) use at baseline. BMI, diabetes mellitus, hypertension and high cholesterol are often considered aspects of metabolic syndrome. These variables were included in our model as previous research has indicated there may be underlying metabolic contributions to the development of comorbid CVD in individuals with OA [8]. NSAID use was also chosen as a possible confounder as it too has been indicated as a possible contributor to increased CVD risk in individuals with OA [7].

\section{Statistical analysis}

Descriptive statistics were calculated for all participants in the final analytic sample. Logistic regression models were used to model the odds of incident CVD. These population-averaged models for non-normal (binomial) measures were fit to each of the three physical function measure analyses. First, unadjusted odds ratios (OR) and 95\% confidence intervals $(\mathrm{CI})$ were computed to examine associations of sxKOA, baseline function, and worsening function with incident CVD. Second, multivariable models that jointly examined associations of sxKOA, baseline function and worsening function with incident CVD (Model 1) were conducted. Third, multivariable models that included Model 1 variables along with relevant demographic and health covariates (Model 2) were conducted. Models 1 and 2 were assessed for the interaction of sxKOA with physical function (both the baseline measure and the follow-up worsening indicator) at the 0.05 significance level. All statistical computations were performed using SAS, version 9.4 (Cary, NC).

\section{Results}

Participant characteristics are summarized in Table 1. Almost a quarter of our sample consisted of the Enrichment cohort, and overall mean time to follow-up was $5.9 \pm 1.2$ years. The sample comprised $63.6 \%$ women, 27.8\% African Americans, and 25.9\% with less than a high school education, while mean age of participants was 59.5 \pm 9.5 years. The mean BMI was $29.3 \pm 6.0 \mathrm{~kg} / \mathrm{m}^{2}$, with over three quarters of participants being overweight $(38.2 \%)$ or obese $(38.4 \%)$. At baseline, approximately a tenth of participants had diabetes, a third had hypertension, a fifth had high blood cholesterol, and almost a third reported NSAID use. Baseline sxKOA was present for approximately $15 \%$ of participants, and approximately $16 \%$ of participants developed CVD by their first follow-up. Individuals with sxKOA at baseline had 1.50 times the unadjusted odds of incident CVD (95\% CI 1.08-2.08).

\section{HAQ}

Regarding mean HAQ scores, over half of participants (58.6\%) had a score of zero, almost a third (30.8\%) had $0<\mathrm{HAQ}<1$, and about a tenth (10.6\%) had $1 \leq \mathrm{HAQ}$ at baseline. About a third (33.0\%) of participants worsened by 0.22 units or more in mean HAQ score by first follow-up (which corresponds to the minimum clinically important difference), with the remainder staying the same or improving. 
Table 1 Descriptive Characteristics of Study Sample $(N=1709)$

\begin{tabular}{|c|c|}
\hline Characteristic & Mean (SD) or \% \\
\hline \multicolumn{2}{|l|}{ Baseline } \\
\hline$\%$ Original cohort & 75.6 \\
\hline \multicolumn{2}{|l|}{ Demographic } \\
\hline Mean (SD) Age & $59.5(9.5)$ \\
\hline$\%$ Women & 63.6 \\
\hline$\%$ African American & 27.8 \\
\hline$\%$ with $<12$ Years Education & 25.9 \\
\hline \multicolumn{2}{|l|}{ Health Related } \\
\hline Mean (SD) Body Mass Index (kg/m²) & $29.3(6.0)$ \\
\hline Mean (SD) Waist to Hip Ratio & $0.87(0.09)$ \\
\hline \% Hypertension & 32.9 \\
\hline$\%$ Diabetes & 8.7 \\
\hline$\%$ High cholesterol & 20.8 \\
\hline \multicolumn{2}{|l|}{ OA Related } \\
\hline$\%$ Non-steroidal Anti-inflammatory Drug Use & 31.5 \\
\hline \% Symptomatic Osteoarthritis & 15.4 \\
\hline \multicolumn{2}{|l|}{ Function Related } \\
\hline Mean Health Assessment Questionnaire; HAQ (SD) & $0.27(0.47)$ \\
\hline$\% 0=\mathrm{HAQ}$ & 58.6 \\
\hline$\% 0<\mathrm{HAQ}<1$ & 30.8 \\
\hline$\% 1 \leq \mathrm{HAQ}$ & 10.6 \\
\hline Mean (SD) Speed During $8 \mathrm{ft}$. Walk (m/s) & $0.87(0.26)$ \\
\hline Mean (SD) Chair Stand Times (s) & $12.2(4.8)$ \\
\hline$\% \mathrm{Q}^{\mathrm{a}}$ & 20.5 \\
\hline$\%$ Q2 & 23.1 \\
\hline \% Q3 & 23.5 \\
\hline$\%$ Q4 & 27.5 \\
\hline \% Unable to Complete & 5.4 \\
\hline \multicolumn{2}{|l|}{ Follow-up } \\
\hline Mean (SD) years to follow-up & $5.9(1.2)$ \\
\hline$\%$ Worsened HAQ & 33.0 \\
\hline$\%$ Worsened $8 \mathrm{ft}$. Walk speed & 49.6 \\
\hline$\%$ Worsened Chair Stand Time & 42.2 \\
\hline \% Incident Cardiovascular Disease & 15.9 \\
\hline
\end{tabular}

$\mathrm{a}_{5}$ Chair stand time quartlies defined from the non-exposure subsample without sxKOA at baseline: Males: Q1 (time $<=8.4 \mathrm{~s})$, Q2 $(8.4 \mathrm{~s}<$ time $<=10.2 \mathrm{~s})$ ), Q3 $(10.2 \mathrm{~s}<$ time < = 12.9 s), Q4 (12.9 s < time)"; Females: Q1 (time < =9.0 s), Q2 (9.0 s $<$ time < =11.3 s), Q3 (11.3 s< time <=14.1 s), Q4 (14.1 s < time)

$S D$ standard deviation, $O A$ Osteoarthritis

There were no significant interactions of sxKOA with baseline or worsening HAQ scores, so overall main effects are reported (Table 2). In unadjusted analyses, participants with baseline HAQ scores >0 had 30-90\% higher odds of incident CVD compared to those with $\mathrm{HAQ}=0$; those with worsening HAQ scores over time had 2.5 times greater odds of incident CVD. In multivariable Model 1, baseline HAQ score $\geq 1$ and worsening HAQ continued to have significantly greater odds of incident CVD, but sxKOA was no longer significantly associated with CVD risk in this model. These associations were similar in the fully adjusted model (Model 2), in which age and self-reported diabetes were also associated with incident CVD.

\section{Chair stands}

For chair stands, $5.4 \%$ of participants were unable to complete the test at baseline, and the quartile cutoff distribution (fastest to slowest times) was 20.5\%, 23.1\%, 23.5 and $27.5 \%$. About $42 \%$ of participants worsened by moving up a quartile (or becoming unable) in chair stand performance at follow-up, with the remaining staying the same or improving.

There were no significant interactions of sxKOA with baseline or worsening chair stand time and so overall main effects are reported (Table 3). Compared to participants in the lowest quartile (Q1) of chair stand time, those in higher quartiles (Q2, Q3, and Q4) and those unable to complete the test had significantly elevated unadjusted odds of incident CVD; worsening chair stand performance over time was also associated with increased incident CVD. In multivariable Model 1, similar associations with incident CVD remained for baseline chair stand quartiles and worsening chair stand time, but sxKOA was no longer significantly associated with incident CVD risk in this model. These associations were similar in the fully adjusted model (Model 2), although inability to complete chair stands was no longer significantly associated with incident CVD risk; sex and diabetes were also associated with increased CVD risk.

\section{8-foot walk}

The mean baseline gait speed during the 8 - $\mathrm{ft}$ walk was $0.87 \mathrm{~m} / \mathrm{s}(\mathrm{SD}=0.26)$. About half $(49.6 \%)$ of participants worsened by decreasing $0.1 \mathrm{~m} / \mathrm{s}$ or more in mean gait speed by first follow-up. The measure of baseline gait speed showed a significant interaction with baseline sxKOA status, so effects of baseline gait speed are shown separately for participants without and with sxKOA (Table 4). Results for baseline gait speed are presented in units of $0.3 \mathrm{~m} / \mathrm{s}$ difference; this was selected because it approximated 1 standard deviation for the distribution of baseline gait speeds. For participants without sxKOA, unadjusted odds of incident CVD were higher for those with a slower gait speed at baseline. In contrast, for participants with sxKOA, baseline gait speed was not associated with incident CVD. In unadjusted analyses, there was no association between worsening gait speed and incident CVD. 
Table 2 Unadjusted and Adjusted Associations of sXKOA and HAQ Scores with Incident CVD

\begin{tabular}{|c|c|c|c|}
\hline \multirow[t]{2}{*}{ Variable } & Unadjusted & Model $1^{a}$ & Model $2^{a}$ \\
\hline & OR $(95 \% \mathrm{Cl})$ & OR $(95 \% \mathrm{Cl})$ & OR $(95 \% \mathrm{Cl})$ \\
\hline sxKOA vs. no sxKOA at baseline & $1.50(1.08,2.08)$ & $1.14(0.80,1.61)$ & $1.10(0.76,1.61)$ \\
\hline Baseline $0<\mathrm{HAQ}<1$ vs. $\mathrm{HAQ}=0$ & $1.29(0.97,1.72)$ & $1.17(0.87,1.57)$ & $1.08(0.79,1.47)$ \\
\hline Baseline $1 \leq \mathrm{HAQ}$ vs. $\mathrm{HAQ}=0$ & $1.94(1.32,2.85)$ & $1.91(1.27,2.86)$ & $1.82(1.18,2.79)$ \\
\hline Worsened HAQ vs. unchanged or improved & $2.51(1.93,3.27)$ & $2.49(1.90,3.25)$ & $2.35(1.79,3.10)$ \\
\hline Age: 1 year increase & & & $1.02(1.00,1.03)$ \\
\hline Gender: Female vs. Male & & & $1.28(0.91,1.80)$ \\
\hline Race: Black vs. White & & & $1.13(0.83,1.54)$ \\
\hline Education: <HS vs. HS or greater & & & $0.89(0.65,1.24)$ \\
\hline Cohort: Enrichment vs. Original & & & $0.96(0.69,1.33)$ \\
\hline BMI: $1 \mathrm{~kg} / \mathrm{m}^{2}$ increase & & & $0.98(0.95,1.00)$ \\
\hline WHR: 0.1 unit increase & & & $1.15(0.98,1.36)$ \\
\hline Diabetes vs. not & & & $1.84(1.21,2.80)$ \\
\hline Hypertension vs. not & & & $1.10(0.81,1.48)$ \\
\hline High cholesterol vs. not & & & $1.03(0.74,1.44)$ \\
\hline NSAIDs vs. not & & & $1.27(0.94,1.71)$ \\
\hline
\end{tabular}

$\mathrm{Cl}$ confidence interval, sXKOA symptomatic knee osteoarthritis, HAQ health assessment questionnaire, HS high school, BMI body mass index, WHR waist-to-hip ratio, NSAIDs non-steroidal anti-inflammatory drugs

${ }^{a}$ Models 1 and 2 are adjusted for all variables with data listed in the column

Table 3 Unadjusted and Adjusted Associations of sxKOA and Chair Stands with Incident CVD

\begin{tabular}{|c|c|c|c|}
\hline \multirow[t]{2}{*}{ Variable } & Unadjusted & Model $1^{a}$ & Model $2^{a}$ \\
\hline & OR $(95 \% \mathrm{Cl})$ & OR $(95 \% \mathrm{Cl})$ & OR $(95 \% \mathrm{Cl})$ \\
\hline sxKOA vs. no sxKOA at baseline & $1.50(1.08,2.08)$ & $1.28(0.90,1.80)$ & $1.24(0.86,1.79)$ \\
\hline Baseline 5 chair stand time Q2 vs. Q1 ${ }^{* *}$ & $1.77(1.14,2.75)$ & $1.82(1.17,2.83)$ & $1.77(1.13,2.78)$ \\
\hline Baseline 5 chair stand time Q3 vs. Q1 ${ }^{* *}$ & $1.93(1.25,2.98)$ & $2.04(1.31,3.16)$ & $1.79(1.14,2.81)$ \\
\hline Baseline 5 chair stand time Q4 vs. Q1** & $1.85(1.21,2.82)$ & $2.02(1.30,3.15)$ & $1.65(1.02,2.65)$ \\
\hline Baseline 5 chair stand time unable vs. Q1** & $2.50(1.36,4.58)$ & $2.14(1.16,3.98)$ & $1.71(0.89,3.27)$ \\
\hline Worsened 5 Chair stand time vs. unchanged or improved & $1.50(1.15,1.94)$ & $1.58(1.20,2.08)$ & $1.45(1.09,1.93)$ \\
\hline Age: 1 year increase & & & $1.01(1.00,1.03)$ \\
\hline Gender: Female vs. Male & & & $1.46(1.05,2.04)$ \\
\hline Race: Black vs. White & & & $1.11(0.82,1.52)$ \\
\hline Education: <HS vs. HS or greater & & & $0.95(0.68,1.31)$ \\
\hline Cohort: Enrichment vs. Original & & & $1.06(0.77,1.47)$ \\
\hline BMI: 1 kg/m² increase & & & $0.98(0.96,1.01)$ \\
\hline WHR: 0.1 unit increase & & & $1.16(0.99,1.36)$ \\
\hline Diabetes vs. not & & & $1.91(1.26,2.89)$ \\
\hline Hypertension vs. not & & & $1.13(0.84,1.52)$ \\
\hline High cholesterol vs. not & & & $1.03(0.74,1.44)$ \\
\hline NSAIDs vs. not & & & $1.30(0.97,1.74)$ \\
\hline
\end{tabular}

Cl confidence interval, Q quarter, sxKOA symptomatic knee osteoarthritis, HS high school, BMI body mass index, WHR waist-to-hip ratio, NSAIDs non-steroidal anti-inflammatory drugs

${ }^{a}$ Models 1 and 2 are adjusted for all variables with data listed in the column 
Table 4 Unadjusted and Adjusted Associations of sxKOA and 8-Foot Walk with Incident CVD

\begin{tabular}{|c|c|c|c|}
\hline \multirow[t]{2}{*}{ Variable } & \multirow{2}{*}{$\begin{array}{l}\text { Unadjusted } \\
\text { OR (95\% Cl) }\end{array}$} & \multirow{2}{*}{$\begin{array}{l}\text { Model } 1^{\text {a }} \\
\text { OR }(95 \% \text { Cl) }\end{array}$} & \multirow{2}{*}{$\begin{array}{l}\text { Model } 2^{\text {a }} \\
\text { OR (95\% Cl) }\end{array}$} \\
\hline & & & \\
\hline Baseline $0.3 \mathrm{~m} / \mathrm{s}$ (1SD) decrease in mean $8 \mathrm{ft}$. walk speed among those without sxKOA & $1.46(1.23,1.74)$ & $1.64(1.35,1.99)$ & $1.46(1.17,1.82)$ \\
\hline Baseline $0.3 \mathrm{~m} / \mathrm{s}$ (1SD) decrease in mean $8 \mathrm{ft}$. walk speed among those with sxKOA & $0.91(0.62,1.33)$ & $0.99(0.67,1.47)$ & $0.90(0.59,1.36)$ \\
\hline Worsened $8 \mathrm{ft}$. walk vs. unchanged or improved & $1.12(0.87,1.45)$ & $1.53(1.15,2.04)$ & $1.47(1.07,2.01)$ \\
\hline Age: 1 year increase & & & $1.01(0.99,1.03)$ \\
\hline Gender: Female vs. Male & & & $1.35(0.96,1.91)$ \\
\hline Race: Black vs. White & & & $1.05(0.77,1.44)$ \\
\hline Education: <HS vs. HS or greater & & & $0.92(0.66,1.27)$ \\
\hline Cohort: Enrichment vs. Original & & & $1.10(0.79,1.54)$ \\
\hline BMI: $1 \mathrm{~kg} / \mathrm{m}^{2}$ increase & & & $0.98(0.96,1.01)$ \\
\hline WHR: 0.1 unit increase & & & $1.15(0.98,1.36)$ \\
\hline Diabetes vs. not & & & $1.80(1.19,2.71)$ \\
\hline Hypertension vs. not & & & $1.11(0.83,1.49)$ \\
\hline High cholesterol vs. not & & & $1.09(0.79,1.52)$ \\
\hline NSAIDs vs. not & & & $1.34(1.00,1.80)$ \\
\hline
\end{tabular}

In multivariable Model 1, slower baseline gait speed remained associated with incident CVD only for those without sxKOA. Worsening gait speed was also associated with incident CVD in Model 1. These associations were similar in the fully adjusted Model 2, in which NSAID use and self-reported diabetes were also associated with incident CVD.

\section{Discussion}

In this analysis, we examined the unique contributions of sxKOA, baseline function and worsening of function with incident CVD risk. With respect to sxKOA, we found significant associations with incident CVD risk in unadjusted analyses, but not in multivariable analyses adjusting for functional variables. There were significant associations of functional variables with incident CVD risk, and in particular, worsening of function variables over time was consistently associated with CVD risk in multivariable models. The association of one function variable, 8-ft walk, differed between those with and without sxKOA, being important only in the latter group.

The finding of a significant bivariate association of sxKOA with CVD risk is in agreement with results of prior studies [5, 10-12, 25]. Importantly, the longitudinal nature of this association supports that sxKOA predicts development of future CVD. In multivariable analyses including function variables, even prior to adjustment for other confounders, sxKOA was no longer significantly associated with CVD risk. This confirms another recent longitudinal study in which the association of number of joints with OA with CVD risk was explained by self-reported difficulty walking at baseline [12]. These studies indicate that function is at least one key contributor to CVD risk among individuals with sxOA. Knowledge of this underlying mechanism is extremely important, as it points to a potential intervention approach. In particular, physical activity programs can significantly improve functional outcomes among individuals with sxKOA [26] and therefore may confer an important benefit regarding downstream CVD risk, particularly since physical activity also improves a number of metabolic factors leading to CVD.

There were a number of interesting findings regarding associations of functional measures with CVD risk. First, it is notable that all of the function measures (with the exception of 8-ft walk for people with sxKOA) were associated with increased CVD risk, suggesting that both performance-based measures and self-report measures (e.g., HAQ) may be helpful markers of CVD risk; this adds to prior studies that have shown positive associations with self-report function measures. Second, even when baseline function and change in function were included in the same model, change in function consistently remained significantly associated with incident CVD risk. This also adds to prior studies, which have focused on functional status at a single time point. These results illustrate the importance of functional decline in the prediction of CVD, regardless of baseline functional status, and further illustrates the importance of physical activity programs that can slow the progression of functional decline. Third, functional measures (particularly change in function over time) continued to have 
significant associations with CVD risk even in fully adjusted models that included a number of factors related to metabolic syndrome (e.g, BMI, WHR, diabetes). This is important since metabolic syndrome has been another proposed mechanism underlying the association between OA and CVD [7, 8]. Kendzerska et al. also found that self-reported walking-related disability continued to predict CVD risk even when adjusting for BMI and other metabolic factors [12]. This evidence supports that function plays a unique role in the relationships between sxKOA and CVD risk. Fourth, there was an interaction between sxKOA and the 8 - $\mathrm{ft}$ walk variable: those without sxKOA who had worse baseline 8 - $\mathrm{ft}$ walk speed were at a greater risk of developing incident CVD, but for those with sxKOA, baseline $8-\mathrm{ft}$ walk performance was not a predictor of incident CVD risk. We hypothesize this result may be due to the fact that our sxKOA group had worse baseline walking speeds than the non-OA group at baseline $(.75 \mathrm{~m} / \mathrm{s}$ vs. $89 \mathrm{~m} / \mathrm{s})$, which confirms prior studies [27, 28]. Since walking speed was low overall among those with sxKOA, the variability within that lower range may not have been associated with differential CVD risk.

The association of between baseline chair stand time categories and increased CVD risk was non-linear. Relative to participants in the best baseline chair stand category (Q1), odds of CVD risk were elevated more for middle categories (Q2, Q3) than for those in the worst categories (Q4, unable to complete). However, the odds ratios were still relatively similar (1.65-1.79 in fully adjusted models). The lack of completely linear relationship may be due to the relatively small number of participants in each baseline chair stand group.

Strengths of this study include a community-based cohort including African American and Caucasian men and women, the use of both self-reported and performancebased measures of physical function, the longitudinal approach, and the inclusion of worsening variables for physical function measures. However, there are several limitations to note. First, CVD and function measures at follow-up were assessed at a single time point; therefore, we could not ascertain the specific time of occurrence, and it is possible that functional decline occurred after a CVD diagnosis or event within the follow-up period. Second, incident CVD was assessed via self-report. Although this method allows data for large samples to be obtained readily and cost-effectively, this can result in less accurate representation of true CVD. Strategies were employed in data collection in order to maximize self-report accuracy of CVD. These included impartial and standardized phrasing of the National Health Interview Survey question, ensuring that respondents understood the question completely, and that adequate amount of time for recall was given. However, there may still be instances of inaccurate reporting (either over-reporting or under-reporting).
Third, the incident CVD variable definition differed somewhat between cohorts, with angina and congestive heart only being included for the Enrichment Cohort. Fourth, some data were missing due to loss-to-follow-up, and it is possible that individuals with missing follow-up data were less healthy and had more functional limitations than those with complete data.

\section{Conclusion}

Overall, our study indicates that function is a key contributor to the association between symptomatic knee osteoarthritis and cardiovascular disease risk. Further, worsening of function over time seems to have a particularly important role. Physical activity and structured exercise programs can substantially improve function and are already key recommendations for OA management [26]. Unfortunately, many people with symptomatic knee osteoarthritis remain physically inactive [29], placing them at risk for functional loss, and perhaps subsequent elevation of cardiovascular disease risk as a result. These results further elevate the importance of efforts to enhance physical activity among individuals with symptomatic knee osteoarthritis and also highlight the importance of regular physical function assessment.

\section{Abbreviations \\ BMI: Body Mass Index; CCA: Complete case analysis; Cl: Confidence interval; CVD: Cardiovascular disease; HAQ: Health Assessment Questionnaire; JoCo OA: Johnston County Osteoarthritis Project; K-L: Kellgren Lawrence; NSAID: Non-steroidal Anti-Inflammatory Drug; OA: Osteoarthritis; OR: Odds ratio; Q: Quartile; SD: Standard deviation; SxKOA: Symptomatic knee osteoarthritis; WHR: Waist to Hip Ratio}

\section{Acknowledgements}

We are thankful to the participants and staff in the Johnston County Osteoarthritis Project. Their efforts and dedication to the project made this research possible.

\section{Funding}

JoCo OA is supported by the Centers for Disease Control and Prevention / Association of Schools of Public Health cooperative agreements S043, S1734 and S3486 and U01DP003206 and U01DP006266. Further funding was supplied by NIAMS Multipurpose Arthritis and Musculoskeletal Disease Center grant 5-P60-AR3070, NIAMS Multidisciplinary Clinical Research Center grant 5 P60 AR49465-03 and P60AR30701, and NIA 5-T35-AG038047-07 UNC-CH Summer Research in Aging for Medical Students (MSTAR) and the Center for Health Services Research in Primary Care, Durham VA Health Care System (CIN 13-410). The findings and conclusions in this report are those of the authors and do not necessarily represent the official position of the Centers for Disease Control and Prevention. The authors retained full independence with respect to design of the study, data collection, analysis, interpretation of data and writing the manuscript.

\section{Availability of data and materials}

The datasets generated and/or analyzed during the current study are not publicly available due to feasibility but are available (in de-identified form) from the corresponding author on reasonable request.

\section{Authors' contributions}

MC, CA, AT and KDA contributed to initial planning of these secondary analyses and drafting of the manuscript. CA and RC contributed to plans for and conduct of statistical analyses. LFC, RC, YMG, JMJ, AEN, and JR contributed to the design and conduct of the parent study. All authors reviewed, edited and approved the final manuscript. 


\section{Ethics approval and consent to participate}

The study was approved by the Institutional Review Board of the University of North Carolina at Chapel Hill and the Centers for Disease Control and Prevention. All study participants provided written informed consent.

\section{Consent for publication}

Not applicable

\section{Competing interests}

The authors declare that they have no competing interests.

\section{Publisher's Note}

Springer Nature remains neutral with regard to jurisdictional claims in published maps and institutional affiliations.

\section{Author details}

${ }^{1}$ College of Medicine, Northeast Ohio Medical University, 4209 OH-44, Rootstown, OH 44272, USA. ${ }^{2}$ Thurston Arthritis Research Center, University of North Carolina at Chapel Hill, 3300 Thurston Bldg., CB\# 7280, Chapel Hill, NC 27599, USA. ${ }^{3}$ Department of Medicine, University of North Carolina at Chapel Hill, 125 MacNider Hall CB\# 7005, Chapel Hill, NC 27599, USA. ${ }^{4}$ Injury Prevention Research Center, University of North Carolina at Chapel Hill, CB\# 7505, 137 E Franklin St, Chapel Hill, NC 27599, USA. ${ }^{5}$ Department of Epidemiology, University of North Carolina at Chapel Hill, 170 Rosenau Hall, CB\#7400, Chapel Hill, NC 27599, USA. ${ }^{6}$ Department of Radiology, University of North Carolina at Chapel Hill, 2006 Old Clinic, CB, Chapel Hill, NC \#7510, USA. ${ }^{7}$ Center for Health Services Research in Primary Care, Department of Veterans Affairs Healthcare System, 508 Fulton Street, Durham, NC, USA.

\section{Received: 17 July 2018 Accepted: 19 October 2018}

\section{Published online: 10 November 2018}

\section{References}

1. Cross M, Smith E, Hoy D, Nolte S, Ackerman I, Fransen M, Bridgett L, Williams S, Guillemin F, Hill CL, et al. The global burden of hip and knee osteoarthritis: estimates from the global burden of disease 2010 study. Ann Rheum Dis. 2014;73(7):1323-30.

2. Oiestad BE, White DK, Booton R, Niu J, Zhang Y, Torner J, Lewis CE, Nevitt M, LaValley M, Felson DT. Longitudinal course of physical function in people with symptomatic knee osteoarthritis: data from the multicenter osteoarthritis study and the osteoarthritis initiative. Arthritis Care Res (Hoboken). 2016;68(3):325-31.

3. White DK, Zhang Y, Niu J, Keysor JJ, Nevitt MC, Lewis CE, Torner JC, Neogi T. Do worsening knee radiographs mean greater chances of severe functional limitation? Arthritis Care Res (Hoboken). 2010;62(10):1433-9.

4. Hall AJ, Stubbs B, Mamas MA, Myint PK, Smith TO. Association between osteoarthritis and cardiovascular disease: systematic review and metaanalysis. Eur J Prev Cardiol. 2016;23(9):938-46.

5. Veronese N, Trevisan C, De Rui M, Bolzetta F, Maggi S, Zambon S, Musacchio E, Sartori L, Perissinotto E, Crepaldi G, et al. Association of Osteoarthritis with Increased Risk of cardiovascular diseases in the elderly: findings from the Progetto Veneto Anziano study cohort. Arthritis Rheumatol. 2016;68(5):1136-44.

6. Rahman MM, Kopec JA, Anis AH, Cibere J, Goldsmith CH. Risk of cardiovascular disease in patients with osteoarthritis: a prospective longitudinal study. Arthritis Care Res (Hoboken). 2013;65(12):1951-8.

7. Fernandes GS, Valdes AM. Cardiovascular disease and osteoarthritis: common pathways and patient outcomes. Eur J Clin Investig. 2015;45(4):405-14.

8. Le Clanche S, Bonnefont-Rousselot D, Sari-Ali E, Rannou F, Borderie D. Interrelations between osteoarthritis and metabolic syndrome: a common link? Biochimie. 2016;121:238-52.

9. Schieir $\mathrm{O}$, Hogg-Johnson S, Glazier RH, Badley EM. Sex variations in the effects of arthritis and activity limitation on first heart disease event occurrence in the Canadian general population: results from the longitudinal National Population Health Survey. Arthritis Care Res (Hoboken). 2016;68(6):811-8.

10. King L, Kendzerska T, Hawker G. The Relationship Between Osteoarthritis and Cardiovascular Disease: Results from a Population-Based Cohort. American College of Rheumatology Annual Scientific Meeting. 2015;67:954.

11. Hawker GA, Croxford R, Bierman AS, Harvey PJ, Ravi B, Stanaitis I, Lipscombe LL. All-cause mortality and serious cardiovascular events in people with hip and knee osteoarthritis: a population based cohort study. PLoS One. 2014; 9(3):e91286.
12. Kendzerska T, Juni P, King LK, Croxford R, Stanaitis I, Hawker GA. The longitudinal relationship between hand, hip and knee osteoarthritis and cardiovascular events: a population-based cohort study. Osteoarthr Cartil. 2017:25(11):1771-80

13. Jordan JM, Helmick CG, Renner JB, Luta G, Dragomir AD, Woodard J, Fang F, Schwartz TA, Abbate LM, Callahan LF, et al. Prevalence of knee symptoms and radiographic and symptomatic knee osteoarthritis in African Americans and Caucasians: the Johnston County osteoarthritis project. J Rheumatol. 2007;31(4):172-80.

14. Allison PD. Quantitative applications in the social sciences: missing data. Thousand Oaks, CA: Sage Publications Ltd; 2002.

15. Bruce B, Fries JF. The Stanford health assessment questionnaire: dimensions and practical applications. Health Qual Life Outcomes. 2003;1:20.

16. Elliott AL, Kraus VB, Fang F, Renner JB, Schwartz TA, Salazar A, Huguenin T, Hochberg MC, Helmick CG, Jordan JM. Joint-specific hand symptoms and self-reported and performance-based functional status in African Americans and Caucasians: the Johnston County osteoarthritis project. Ann Rheum Dis. 2007;66(12):1622-6.

17. Bijsterbosch J, Scharloo M, Visser AW, Watt I, Meulenbelt I, Huizinga TW, Kaptein AA, Kloppenburg M. Illness perceptions in patients with osteoarthritis: change over time and association with disability. Arthritis Rheum. 2009:61(8):1054-61.

18. Kosinski M, Zhao SZ, Dedhiya S, Osterhaus JT, Ware JE Jr. Determining minimally important changes in generic and disease-specific health-related quality of life questionnaires in clinical trials of rheumatoid arthritis. Arthritis Rheum. 2000;43(7):1478-87.

19. Dobson F, Hinman RS, Roos EM, Abbott JH, Stratford P, Davis AM, Buchbinder R, Snyder-Mackler L, Henrotin Y, Thumboo J, et al. OARSI recommended performance-based tests to assess physical function in people diagnosed with hip or knee osteoarthritis. Osteoarthr Cartil. 2013;21(8):1042-52.

20. Guralnik JM, Simonsick EM, Ferrucci L, Glynn RJ, Berkman LF, Blazer DG, Scherr PA, Wallace RB. A short physical performance battery assessing lower extremity function: association with self-reported disability and prediction of mortality and nursing home admission. J Gerontol. 1994;49(2):M85-94.

21. Cleveland RJ, Schwartz TA, Renner J, Jordan JM, Callahan LF. Physical function and mortality: the Johnston County osteoarthritis project. Boston MA: American college of rheumatology annual scientific meeting; 2014.

22. Purser JL, Golightly YM, Feng Q, Helmick CG, Renner JB, Jordan JM. Association of slower walking speed with incident knee osteoarthritisrelated outcomes. Arthritis Care Res (Hoboken). 2012;64(7):1028-35.

23. Hardy SE, Perera S, Roumani YF, Chandler JM, Studenski SA. Improvement in usual gait speed predicts better survival in older adults. J Am Geriatr Soc. 2007:55(11):1727-34.

24. Middleton A, Fritz SL, Lusardi M. Walking speed: the functional vital sign. J Aging Phys Act. 2015;23(2):314-22.

25. Ong KL, Wu BJ, Cheung BM, Barter PJ, Rye KA. Arthritis: its prevalence, risk factors, and association with cardiovascular diseases in the United States, 1999 to 2008. Ann Epidemiol. 2013;23(2):80-6.

26. Roddy E, Zhang W, Doherty M. Aerobic walking or strengthening exercise for osteoarthritis of the knee? A systematic review. Ann Rheum Dis. 2005; 64(4):544-8.

27. Pisters MF, Veenhof C, van Dijk GM, Heymans MW, Twisk JW, Dekker J. The course of limitations in activities over 5 years in patients with knee and hip osteoarthritis with moderate functional limitations: risk factors for future functional decline. Osteoarthr Cartil. 2012;20(6):503-10.

28. Queen RM, Sparling TL, Schmitt D. Hip, knee, and ankle osteoarthritis negatively affects mechanical energy exchange. Clin Orthop Relat Res. 2016; 474(9):2055-63.

29. Song J, Hochberg MC, Chang RW, Hootman JM, Manheim LM, Lee J, Semanik PA, Sharma L, Dunlop DD, Osteoarthritis Initiative I. Racial and ethnic differences in physical activity guidelines attainment among people at high risk of or having knee osteoarthritis. Arthritis Care Res (Hoboken). 2013;65(2):195-202. 